\title{
Effects of chronic heart disease on skeletal muscle fiber size
}

\author{
A.C. Mattiello-Sverzut ${ }^{1}$, \\ L. Chimelli², S. Teixeira ${ }^{1}$, \\ M. Pierre ${ }^{3}$ \\ and L. Oliveira ${ }^{3}$
}

\author{
1'Departamento de Biomecânica, Medicina e Reabilitação do Aparelho Locomotor, \\ Faculdade de Medicina de Ribeirão Preto, Universidade de São Paulo, \\ Ribeirão Preto, SP, Brasil \\ ${ }^{2}$ Departamento de Patologia, Faculdade de Medicina, \\ Universidade Federal do Rio de Janeiro, Rio de Janeiro, RJ, Brasil \\ ${ }^{3}$ Departamento de Estatística, Universidade Federal de São Carlos, \\ São Carlos, SP, Brasil
}

\section{Correspondence}

L. Chimelli

Serviço de Anatomia Patológica

Hospital Universitário, UFRJ

21941-590 Rio de Janeiro, RJ

Brasil

Fax: +55-21-526-2450

E-mail: chimelli@hucff.ufrj.br

Publication supported by FAPESP.

Received August 1, 2003

Accepted August 31, 2004

\section{Abstract}

Size changes in muscle fibers of subjects with chronic heart disease (CHD) have been reported, although a consensus has not been achieved. The aims of the present study were to investigate a possible association between CHD and fiber size changes in the brachial biceps compared to subjects without heart disease. Forty-six muscle samples were obtained in autopsies of individuals (13 to 84 years) without neuromuscular disorders, 19 (10 males and 9 females) with, and 27 (14 males and 13 females) without CHD. In all cases muscle sections were stained with hematoxylin and eosin and processed for the visualization of myofibrillar ATPase activity. The lesser diameter of type 1 and type 2 fibers was obtained tracing their outlines (at least 150 fibers of each type per sample) onto an image analyzer connected to a computer. The results were analyzed statistically comparing males and females with and without CHD. Type 1 fiber mean lesser diameters were 51.51 and $54.52 \mu \mathrm{m}$ in males (normal range $34-71 \mu \mathrm{m}$ ) and 45.65 and $55.42 \mu \mathrm{m}$ in females (normal range $34-65 \mu \mathrm{m}$ ) without and with CHD, respectively; type 2 fibers measured 54.31, 58.23, 41.15, and $49.57 \mu \mathrm{m}$, respectively (normal range 36-79 $\mu \mathrm{m}$ for males and 32-59 $\mu \mathrm{m}$ for females). No significant difference in fiber size was detected in 24 males with and without CHD, while in 22 females there was a significant increase in size in those with cardiomyopathy. We concluded that CHD does not determine significant changes in fiber size. However, in females, there is some hypertrophy which, despite within normal range, may reflect morphologic heterogeneity of the sample, or the daily life activities in the upper limbs as a compensatory mechanism to fatigability that affect predominantly the lower limbs in subjects with CHD.

\section{Introduction}

In 1972, Shafiq et al. (1) reported a histopathological study of skeletal muscle in patients with heart disease. After this first de-
Key words

- Morphometry

- Brachial biceps

- Chronic heart disease

- Post mortem muscle

- Type 1 fiber hypertrophy

- Type 2 fiber hypertrophy ..................... scription several investigators demonstrated histologic and/or electromyographic abnormalities in the skeletal muscle of patients with chronic heart disease (CHD, 2-8). However, no consensus exists as to whether there 
is type 1 and/or 2 fiber atrophy (9-13) or whether there exists any degree of fiber atrophy (14). The aim of the present study was to compare the size of skeletal muscle fibers obtained at autopsy from subjects with CHD to the size of fibers obtained from patients without heart disease.

\section{Subjects and Methods}

Muscle samples were obtained at autopsy, 3 to $9 \mathrm{~h}$ after death, from the belly of the brachial biceps of 46 subjects ( $22 \mathrm{fe}$ males and 24 males) ranging in age from 13 to 84 years (mean female age: $45.6 \pm 19$ years; mean male age: $51.8 \pm 18.1$ years). Nineteen samples (10 males, mean age 51.8 \pm 18.8 , and 9 females, mean age $64.2 \pm 10$ ) were from subjects with chronic cardiomyopathy confirmed at autopsy. Twenty-seven samples (14 males, mean age $52.8 \pm 16.4$, and 13 females, mean age $32.5 \pm 11$ ) were from subjects who had suffered sudden death or who had died after a short acute illness, without involvement of the neuromuscular system, as confirmed morphologically. In all cases, the muscle samples were immediately immersed in liquid nitrogen and oriented in order to obtain transverse sections, which were cut into $10-\mu \mathrm{m}$ thick sections with a cryostat at $-20^{\circ} \mathrm{C}$. Each section was placed on a glass coverslip, stained with hematoxylin and eosin and processed for the visualization of myofibrillar ATPase activity at $\mathrm{pH}$ 9.6, 4.6 and 4.3. The fiber outlines were traced onto an Image Analyzer Kontron, KS300, Carl Zeiss (Oberkochen, Germany) connected to an IBM-PC computer. In each muscle sample, at least 150 fibers of each type were measured to obtain the lesser diameter (type 1 fiber lesser diameter, and type 2 fiber lesser diameter). To facilitate the presentation of the results, the morphometric data were divided into four subgroups according to sex and presence or absence of CHD.

The Ethics Committee of the University
Hospital, School of Medicine of Ribeirão Preto, University of São Paulo, approved this study.

\section{Statistical analysis}

The results were analyzed statistically by the Levene test to identify variance equality and by the Student $t$-test to compare classes. The Mann-Whitney test was used to compare the male and female subgroups in the groups with and without cardiomyopathy. The SPSS software (version 10.0) was used for statistical analysis.

\section{Results and Discussion}

Morphological examinations of hematoxylin and eosin-stained sections showed no structural changes in muscle fibers, except for a variation in fiber size, which did not include angulated fibers or atrophy of fascicles. ATPase-stained sections did not show type grouping.

The most frequent cardiac disorder in the group with CHD was hypertensive cardiopathy, but there was also cardiac failure associated with Chagas' disease, cor pulmonale and chronic ischemic myocardiopathy. In most of the patients of the group without CHD the death was related to hypovolemic or septic shock, due to digestive hemorrhage or acute infections (meningitis, pneumonia, intestinal perfuration) respectively; dissecting aneurism of the aorta, acute myocardial infarct and pulmonary thrombo-embolism were also causes of death in this group.

The mean values of type 1 fiber diameters in subjects without and with CHD, were $51.51 \pm 1.62$ and $54.52 \pm 3.07 \mu \mathrm{m}$ in males and $45.65 \pm 2.08$ and $55.42 \pm 2.04 \mu \mathrm{m}$ in females, respectively. For type 2 fibers, the values were: $54.31 \pm 3.42$ and $58.23 \pm$ $3.37 \mu \mathrm{m}$ in males and $41.15 \pm 1.46$ and 49.57 $\pm 1.17 \mu \mathrm{m}$ in females, respectively (Table 1 ).

The Student $t$-test comparing the type 1 and type 2 classes showed a statistically 
significant difference between males and females $(\mathrm{P}<0.05)$.

Regarding subjects with CHD, class comparison (male, autopsy - without cardiomyopathy, type 1 and type 2 fibers, $\mathrm{N}=14$, and male, autopsy - chronic cardiomyopathy, type 1 and type 2 fibers, $\mathrm{N}=10$ ) showed no significant difference. The results obtained for females showed a statistically significant difference in mean lesser diameter of type 1 and type 2 fibers between subjects with cardiomyopathy $(\mathrm{N}=9)$ and subjects without heart disease $(\mathrm{N}=13 ; \mathrm{P}<0.05$; Figure $1 \mathrm{~A}, \mathrm{~B})$, with higher mean values for females with chronic cardiomyopathy.

The mean lesser diameter obtained for all subjects of both sexes was within the normal values established by Brooke and Engel (15), 40-80 $\mu \mathrm{m}$ for males and 30-70 $\mu \mathrm{m}$ for females, using 103 samples, and by MattielloSverzut et al. (16), 34-71 and 34-65 $\mu \mathrm{m}$ for type 1 fibers, and 36-79 and 32-59 $\mu \mathrm{m}$ for type 2 fibers, in males and females, respectively, using 72 samples. In males with or without chronic cardiomyopathy, type 2 fibers were larger than type 1 fibers, while in females, type 1 fibers were larger than type 2 fibers, as observed by Brooke and Engel (15) in normal muscles. However, among females, subjects with heart disease had a significantly larger mean lesser diameter of type 1 and type 2 fibers than subjects without cardiomyopathy. No literature reports are available about the effects of chronic cardiomyopathy on skeletal muscle in females. For males, the data indicate the presence of mild atrophy, predominantly in type 2 fibers of the vastus lateralis, quadriceps and gastrocnemius muscles of patients with chronic cardiomyopathy $(12,15,17)$. Buller et al. (17) evaluated morphologically and functionally the adductor muscle of the thumb and did not find any size abnormality, not even in strength performance, suggesting only an effect of chronic cardiomyopathy on large muscle groups involved in locomotion. In the brachial biceps muscle, as studied in our cases,
Table 1. Muscle fiber diameters of males (M) and females (F) with and without chronic cardiomyopathy.

\begin{tabular}{lccc}
\hline & Sex & $\begin{array}{c}\text { Without } \\
\text { cardiomyopathy }\end{array}$ & $\begin{array}{c}\text { Chronic } \\
\text { cardiomyopathy }\end{array}$ \\
\hline Type 1 fiber mean & M & $51.51 \pm 1.62$ & $54.52 \pm 3.07$ \\
lesser diameter $(\mu \mathrm{m})$ & $\mathrm{F}$ & $45.65 \pm 2.08$ & $55.42 \pm 2.04^{*}$ \\
Type 2 fiber mean & M & $54.31 \pm 3.42$ & $58.23 \pm 3.37$ \\
lesser diameter $(\mu \mathrm{m})$ & $\mathrm{F}$ & $41.15 \pm 1.46$ & $49.57 \pm 1.17^{*}$
\end{tabular}

Data are reported as means \pm SD for 19 (10 males and 9 females) with, and 27 (14 males and 13 females) without cardiomyopathy.

${ }^{*} P<0.05$ compared to subjects without cardiomyopathy (Student $t$-test).

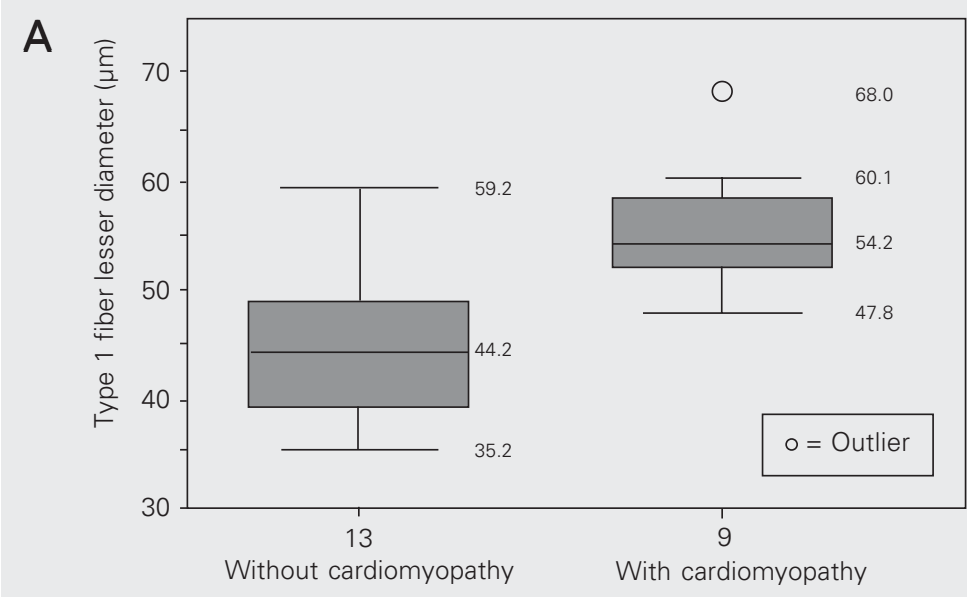

B

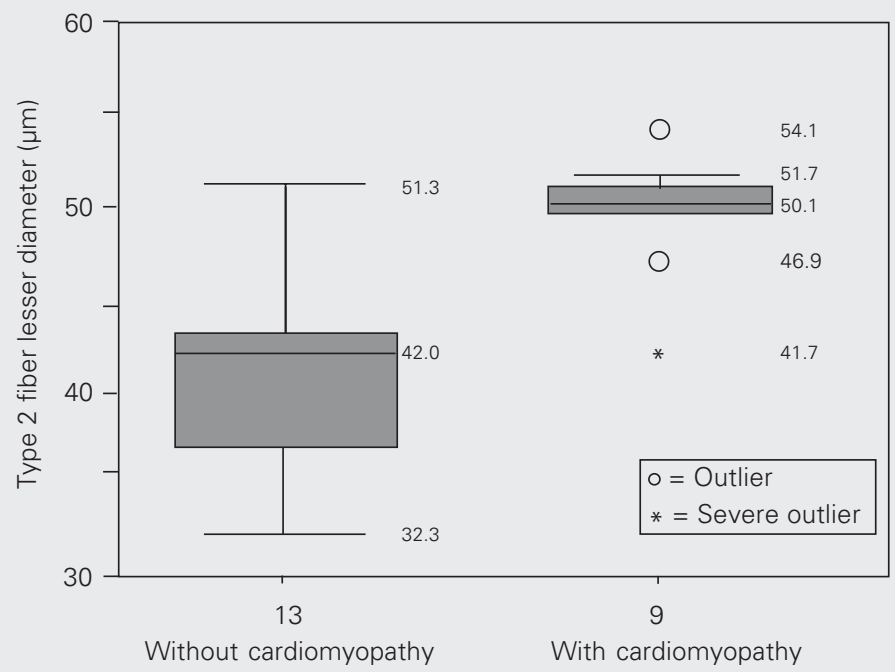

Figure 1. Box plot showing muscle fiber diameters of female autopsy subjects with $(N=9)$ and without $(N=13)$ cardiomyopathy. $A$, Type 1 fibers; $B$, type 2 fibers. ${ }^{*} P<0.05$ compared to subjects without cardiomyopathy (Student $t$-test and when necessary, MannWhitney test). (Fifty percent of the subjects are contained inside the boxes and the central line represents the median. The other $50 \%$ are distributed between the box and the horizontal lines that are above and below the box; the numbers inside the panel represent the values obtained during statistical analysis). 
Caforio et al. (10) observed a reduction of type 1 fibers in one third of the muscle samples from patients with chronic dilated or hypertrophic cardiomyopathy, while the remaining ones presented normal values of type 1 and 2 fibers. In experimental models, both muscle fiber types of the sural triceps of dogs (9) and of the plantaris and sural triceps of rats (18) presented a reduced diameter.

The present results do not confirm the existence of morphological or morphometric changes in upper limb muscles of patients with CHD, as previously reported (1-5). Our findings are intriguing and cannot be fully explained. The increase in size of both fiber types in subjects with chronic cardiomyopathy, more marked in females, may reflect morphologic heterogeneity of the sample, as suggested by Johnson et al. (19), and trophism, as suggested by Shorey and Cleland (20) of the muscles in the human body. This increase may also be determined by effects of daily life on the upper limbs as a compen- satory mechanism for the fatigability and intolerance to exercise predominantly affecting the lower limbs of subjects with chronic cardiomyopathy.

CHD was not associated with significant morphological changes in the brachial biceps muscle of these subjects, although the fibers of females with heart disease were significantly larger than in controls. However, the values obtained were within the normal range established in the literature and in our own observations.

\section{Acknowledgments}

We are grateful to CAPES for providing a Doctoral fellowship for A.C. MattielloSverzut, Department of Pathology, School of Medicine of Ribeirão Preto, USP. The authors also thank M.P.M. Scandar, Department of Pathology, School of Medicine of Ribeirão Preto, USP, for technical assistance.

\section{References}

1. Shafiq SA, Sande MA, Carruthers RR, Killip T \& Milborat AT (1972). Skeletal muscle in idiopathic cardiomyopathy. Journal of Neurological Sciences, 15: 303-320.

2. Isaacs H \& Muncke G (1975). Idiopathic cardiomyopathy and skeletal muscle abnormalities. American Heart Journal, 90: 767-773.

3. Dunnigan A, Pierpont ME, Smith SA, Breningstall G, Benditt DG \& Benson DW (1984). Cardiac and skeletal myopathy associated with cardiac dysrhythmias. American Journal of Cardiology, 53: 731-737.

4. Dunnigan A, Staley NA, Smith SA, Pierpont ME, Judd D, Benditt DG \& Benson Jr DW (1987). Cardiac and skeletal muscle abnormalities in cardiomyopathy: comparison of patients with ventricular tachycardia or congestive heart failure. Journal of the American College of Cardiology, 10: 608-618.

5. Smith ER, Heffernan LP, Sangaland VE, Vaughan LM \& Flamington CS (1976). Voluntary muscle involvement in hypertrophic cardiomyopathy: a study of 11 patients. Annals of Internal Medicine, 85: 566572.

6. Hootsmans WJM \& Meerschwam IS (1971). Electromyography in patients with hypertrophic obstructive cardiomyopathy. Neurology, 21: 810-816

7. Przybojewski JZ, Hoffman H, de Graaf AS, van der Walt JJ, Tiedt FA, O'Kennedy A, Torrington M, Lochner A \& Hewlett R (1981). A study of a family with inherited disease of cardiac and skeletal muscle. Part 1: Clinical electrophysiological and electron microscopic studies. South African Medical Journal, 59: 363-373.

8. Lochner A, Hewlett RH, O'Kennedy A, van der Walt JJ, Tiedt FA, Hoffman H, de Graaf AS, Przybojewski JZ \& Torrington M (1981). A study of a family with inherited disease of cardiac and skeletal muscle. Part 2: Skeletal muscle morphology and mitochondrial oxidative phosphorylation. South African Medical Journal, 59: 453-461.

9. Sabbah HN, Hansen-Smith F, Sharov VG, Kono T, Lesch M, Gengo PJ, Steffen RP, Levine TB \& Goldstein S (1993). Decreased proportion of type I myofibers in skeletal muscle of dogs with chronic heart failure. Circulation, 87: 1729-1737.

10. Caforio ALP, Rossi B, Risaliti R, Siciliano G, Marchetti A, Angelini C, Crea F, Mariani M \& Muratorio A (1989). Type 1 fiber abnormalities in skeletal muscle of patients with hypertrophic and dilated cardiomyopathy: evidence of subclinical myogenic myopathy. Journal of the American College of Cardiology, 14: 1464-1473.

11. Howell S, Maarek JM, Founier M, Sullivan K, Zhan W \& Sieck G (1995). Congestive heart failure: differential adaptation of the diaphragm and latissimus dorsi. Journal of Applied Physiology, 79: 389-397.

12. Mancini DM, Coyle E, Coogan A, Beltz J, Ferraro N, Montain S \& Wilson JR (1989). Contribution of intrinsic skeletal muscle changes to ${ }^{31} \mathrm{P}$ NMR skeletal muscle metabolic abnormalities in patients with chronic heart failure. Circulation, 80: 1338-1346.

13. Sullivan MJ, Green HJ \& Cobb FR (1990). Skeletal muscle biochemistry and histology in ambulatory patients with long-term heart failure. Circulation, 81: 518-527.

14. Brunotte F, Thompson CH, Adamopoulos S, Coats A, Unitt J, Lindsay D, Kaklamanis L, Radda GK \& Rajagopalan B (1995). Rat skeletal muscle metabolism in experimental heart failure: effects of physical training. Acta Physiologica Scandinavica, 154: 439-447. 
15. Brooke $\mathrm{MH}$ \& Engel WK (1969). The histographic analysis of human muscle biopsies with regard to fiber types. 1. Adult male and female. Neurology, 19: 221-234.

16. Mattiello-Sverzut AC, Chimelli L, Moura MSA, Teixeira S \& Mello de Oliveira JA (2003). The effects of aging on brachial biceps muscle fiber size. A morphometrical study in biopsies and autopsies. Arquivos de Neuropsiquiatria, 61: 555-560.

17. Buller NP, Jones D \& Poole-Wilson PA (1991). Direct measurement of skeletal muscle fatigue in patients with chronic heart failure. British Heart Journal, 65: 20-24.
18. Delp MD, Duan C, Mattson JP \& Musch TI (1997). Changes in skeletal muscle biochemistry and histology relative to fiber type in rats with heart failure. Journal of Applied Physiology, 83: 12911299.

19. Johnson MA, Polgar J, Weightman D \& Appleton D (1973). Data on the distribution of fibre types in thirty-six human muscles. An autopsy study. Journal of the Neurological Sciences, 18: 111-129.

20. Shorey CD \& Cleland KW (1988). Morphometric analysis of frozen transverse sections of human skeletal muscle taken post-mortem. Acta Anatomica, 131: 30-34. 